\title{
Lobster eye optics for nano-satellite X-ray monitor
}

\author{
Vladimír Tichýa ${ }^{a}$, Marco Barbera ${ }^{b, c}$, Alfonso Collura ${ }^{c}$, Martin Hromčík ${ }^{d}$, René Hudec ${ }^{e, f}$, \\ Adolf Inneman ${ }^{g}$, Jiří Maršík ${ }^{h}$, Veronika Maršíková ${ }^{g}$, Ladislav Pína $^{i}$, Vojtěch Šimon ${ }^{e}$, \\ Salvatore Varisco $^{c}$ \\ ${ }^{a}$ Department of Control Engineering, Faculty of Electrical Engineering, Czech Technical \\ University in Prague, Karlovo náměstí 13, CZ-12135 Prague 2, Czech Republic \\ ${ }^{b}$ Universitá degli Studi di Palermo, Dipartimento di Fisica, Via Archirafi 36, IT-90123 \\ Palermo, Italy \\ ${ }^{c}$ INAF-Osservatorio Astronomico di Palermo, Piazza del Parlamento 1, IT-90134 Palermo, \\ Italy \\ ${ }^{d}$ Center for Applied Cybernetics, Faculty of Electrical Engineering, Czech Technical \\ University in Prague Karlovo náměstí 13, CZ-12135 Prague 2, Czech Republic \\ ${ }^{e}$ Astronomical Institute, Academy of Sciences of the Czech Republic, Fričova 298, CZ-25165 \\ Ondřejov, Czech Republic \\ ${ }^{f}$ Department of Radioelectronics, Faculty of Electrical Engineering, Czech Technical \\ University in Prague, Technická 2, CZ-16627 Prague 6, Czech Republic \\ ${ }^{g}$ Rigaku Innovative Technologies Europe, s.r.o. Novodvorská 994, CZ-14221 Prague 4, \\ Czech Republic \\ ${ }^{h}$ Division of Precision Mechanics and Optics, Department of Instrumentation and Control \\ Engineering, Faculty of Mechanical Engineering, Czech Technical University, Technická 4, \\ CZ-16607 Prague 6, Czech Republic \\ ${ }^{i}$ Faculty of Nuclear Sciences and Physical Engineering, Czech Technical University in Prague, \\ Břehová 7, CZ-11519 Prague 1, Czech Republic
}

\begin{abstract}
The Lobster eye design for a grazing incidence X-ray optics provides wide field of view of the order of many degrees, for this reason it would be a convenient approach for the construction of space X-ray monitors. In this paper, we compare previously reported measurements of prototype lobster eye X-ray optics called P-25 with computer simulations and discuss differences between the theoretical end experimentally obtained results. Usability of this prototype lobster eye and manufacturing technology for the nano-satellite mission is assessed. The specific scientific goals are proposed.
\end{abstract}

Keywords: Lobster Eye, X-ray optics, X-ray telescope, X-ray sky monitor, X-ray imaging, X-ray

Further author information: (Send correspondence to V. Tichý)

V. Tichý: E-mail: tichyvl1@fel.cvut.cz, Telephone: +420 224357435

M. Barbera: E-mail: barbera@astropa.unipa.it, Telephone: +39 0917571113

A. Collura: E-mail: collura@astropa.unipa.it, Telephone:+39 0917571125

M. Hromčík, E-mail: xhromcik@control.felk.cvut.cz, Telephone: +420224357681

R. Hudec, E-mail: rhudec@gmail.com, Telephone: +420 224352204

A. Inneman, E-mail: Adolf.Inneman@rigaku.com, Telephone: +420 239043333

J. Maršík, E-mail: Jiri.Marsik@rigaku.com, Telephone: +420 239043333

V. Maršíková, E-mail: Veronika.Marsikova@rigaku.com, Telephone: +420 239043333

L. Pína, E-mail: ladislav.pina@fjfi.cvut.cz, Telephone:+420 221912724

V. Šimon, E-mail: vojtech.simon@gmail.com, Telephone: +420 323620347

S. Varisco, E-mail: varisco@astropa.unipa.it, Telephone: +39 0917571122

EUV and X-Ray Optics: Synergy between Laboratory and Space II, edited by René Hudec, Ladislav Pina, Proc. of SPIE Vol. 8076, 80760C - (C) 2011 SPIE · CCC code: 0277-786X/11/\$18 - doi: 10.1117/12.886809 


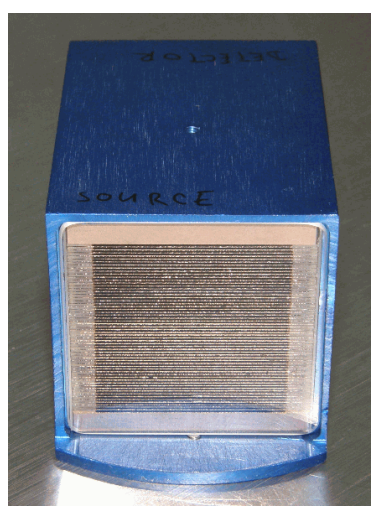

(a)

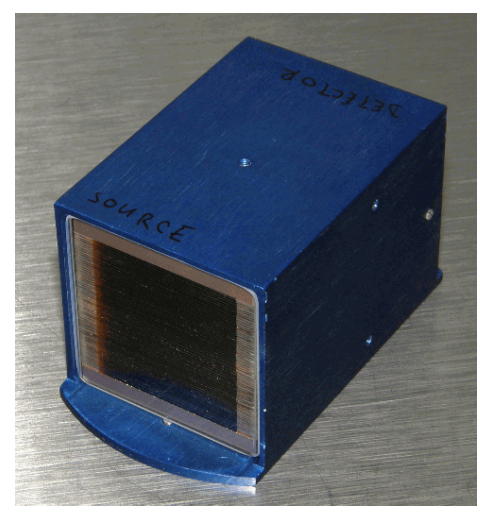

(b)

Figure 1. Prototype lobster eye P-25

\section{INTRODUCTION}

The lobster eye $(\mathrm{LE})^{1,2}$ represents one of the types of grazing incidence reflective X-ray optics. In particular, the Schmidt design ${ }^{1}$ uses two orthogonal sets of reflecting surfaces, each set focuses in one direction.

In comparison with other types of reflective X-ray optics, the main advantage of LE is its large field of view (FOV). Theoretically, the FOV of Schmidt LE can reach around 100 square degrees $^{1}$, so one of the main potential areas of applications lays in future space X-ray monitors ${ }^{3,4}$.

As one of the first steps before bulding larger lobster eye X-ray monitor like ASM/ $R X T E^{5,6}$, nano-satellite lobster eye mission is proposed. Usually, the nomenclature nano-satellite means satellite of dimesions of order of $10 \mathrm{~cm}$ and maximum mass of few kilograms. Especially, the platform called CubeSat ${ }^{11}$ looks to be one of the eaisest approaches for building prototype and experimental space devices. The base unit of CubeSat has dimensions of $10 \times 10 \times 10 \mathrm{~cm}^{3}$ and its maximal mass is limited to $1 \mathrm{~kg}$. Moreover, it is allowed to build specified multiples of the base unit, possible sizes are 0.5, 1.5, 2 and 3 times of the base unit. So, the whole spacecraft can have total dimensions $30 \times 10 \times 10 \mathrm{~cm}^{3}$ and total mass up to $3 \mathrm{~kg}$. This would be sufficient limits for small experimental lobster eye telescope monitoring the selected areas of the sky.

To achieve the best optical performance, the whole lobster eye used in the 3-unit CubeSat should have collecting area around $8 \times 8 \mathrm{~cm}^{2}$ and focal length around $25 \mathrm{~cm}$. So far, few Schmidt LE specimens were manufactured in Rigaku Innovative Technologies Europe, s.r.o. One of these specimens, called P-25 has the corresponding focal length. This specimen has smaller collecting area of $2.4 \times 2.4 \mathrm{~cm}^{2}$, but it is still in order of centimeters. This specimen has undergone X-ray imaging tests to assess the achievable performance of the nano-satellite lobster eye telescope.

\section{LOBSTER EYE P-25}

The prototype lobster eye called P-25 (Fig. 1), manufactured in Rigaku Innovative Techonologies Europe s.r.o., Prague, Czech Republic, consists of two subsystems of mirrors (vertical and horisontal). Each of these subsystems consists of $n=60$ flat reflecting plates made of glass and coated by gold of RMS microroughness $1 \mathrm{~nm}$. The plates (mirrors) have dimensions $24 \times 24 \mathrm{~mm}$ and their thickness is $t=0.1 \mathrm{~mm}$. Average width of the space between the mirrors is $a=0.3 \mathrm{~mm}$. The LE has $f=250 \mathrm{~mm}$ focal length and it is designed for optimal efficiency at $1 \mathrm{keV}$ photon energy.

Performances of this lobster eye were tested in visible light ${ }^{7}$ as well as in X-rays ${ }^{8}$ using 35 meters long X-ray beam-line in INAF-OAPA, Palermo, Italy ${ }^{9,10}$. 


\section{SIMULATION METHOD}

The Schmidt lobster eye has an important property, it consists of two orthogonal subsystems consisting of flat mirrors. Because each of the two subsystems is in principle one-dimensional, its operation can be expressed by one-dimensional matrix. The operation of the whole LE can be expressed as outer product of these two one-dimensional matrices.

Of course, this approach represents an approximation, because rays incoming to the second subsystem of mirrors are not parallel, but deflected by the first subsystem, so grazing angles are slightly different. The advantage of the presented algorithm is that it is simple and fast instead of common ray-tracing methods.

The following methods are separatelly applied to the horisontal and vertical subsystem of mirrors and the resulting image is evaluated as the outer product of results of these two independent computations.

In the simulation program, each mirror is represented by the data structure containing 2-D coordinates of its vertices. The mirror set is represented as an array of these structures.

Detector is represented as an 1-dimensional field of real values, each item of the field represents detected intensity in the corresponding pixel. The detector surface is supposed to be perpendicular to $z$-axis. The detector $z$-position can be set arbitrarily. The pixel size is also stored.

Part of image is due to direct beams, part by one-time reflected beams. Multiplically reflected beams are not taken into accout. Incoming radiation is assumed to be unitary, it means that the direct perpendicular hit of detector pixel causes detection of intensity 1.

In this chapter, the longitudal axis (i.e. optical axis if the whole simulated setup is centered) is labelled $z$. Lateral axis of the one-dimensional system is labelled $b$. Here, symbols $x$ or $y$ are not used to prevent confusion during transformation from two one-dimensional systems to one two-dimensional system.

\subsection{Direct beams}

After initialising the mirror and detector parameters, the effect of the direct beams is simulated.

Simulated situation is shown in Fig. 2. Although in this paper we present only results of simulation for beams

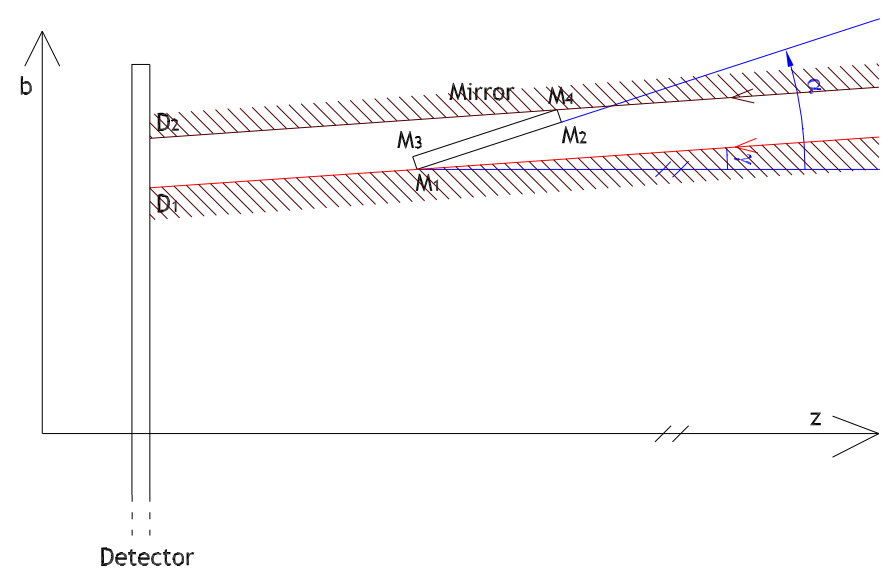

Figure 2. Principle of simulation of direct beams

parallel to the $z$-axis, here the arbitrary slope of incoming rays denoted be $\gamma$ is assumed. Symbol $\alpha$ denotes slope of a mirror relative to the $z$-axis, Both these angles are oriented, positive value means anti-clockwise orientation.

We have found very useful to invert the problem. First, the field representing the detector is filled by $\cos (\alpha)$ to simulate the whole detector being illuminated by radiation of unit flux impacting at angle $\alpha$. Next, the effect of shielding by mirrors is calculated. This calculations are performed as follows. 
First, $b$-positions of the points $\mathbf{D}_{\mathbf{1}}$ and $\mathbf{D}_{\mathbf{2}}$ are evaluated. If $\alpha \geq 0$, then $b$-positions of points $\mathbf{D}_{\mathbf{1}}$ and $\mathbf{D}_{\mathbf{2}}$ can be expressed as

$$
\begin{aligned}
& D_{1}=M_{1 b}-\left(M_{1 z}-D_{z}\right) \tan (\gamma) \\
& D_{2}=M_{4 b}-\left(M_{4 z}-D_{z}\right) \tan (\gamma)
\end{aligned}
$$

If $\alpha<0$, then the points $\mathbf{M}_{\mathbf{2}}$ and $\mathbf{M}_{\mathbf{3}}$ have to be used instead of $\mathbf{M}_{\mathbf{4}}$ and $\mathbf{M}_{\mathbf{1}}$. Symbol $D_{z}$ denotes $z$-position of the detector. Symbol $D_{b}$ denotes $b$-position of the first pixel of the detector.

When the positions of the points $\mathbf{D}_{\mathbf{1}}$ and $\mathbf{D}_{\mathbf{2}}$ are evaluated, they are re-calculated to position on detector in pixels. The values of each fully shaded pixels are set to zero. If some pixel is partially shaded, its value is calculated as the ratio between the length of the iluminated part and the pixel length.

\subsection{Reflected beams}

As the mirrors are considered to be plain (i.e. they are supposed not to be curved; note however that their nonzero thickness will be taken into account), the sufficient task is to calculate positions of reflections of individual mirrors. The situation is described in Fig. 3. Here, $\alpha$ denotes the slope of the mirror relative to $z$-axis, $\beta$ denotes

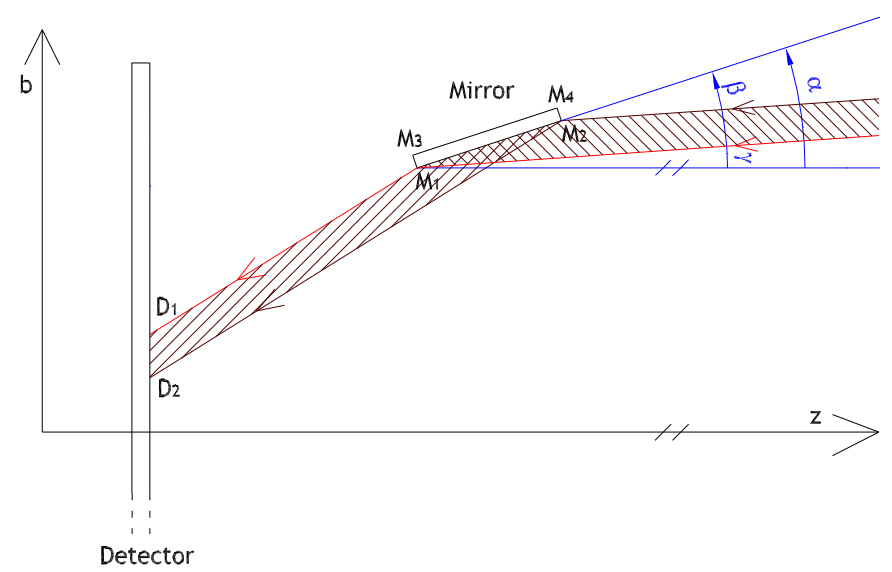

Figure 3. Principle of simulation of reflected beams

the grazing angle. Here we also assume arbitrary slope of the incoming X-rays relative to the $z$-axis denoted by $\gamma$. All named angles are oriented, positive value means anti-clockwise orientation. Symbols $\mathbf{M}_{\mathbf{1}}, \mathbf{M}_{\mathbf{2}}, \mathbf{M}_{\mathbf{3}}, \mathbf{M}_{\mathbf{4}}$ denote vertices of mirror. Points $\mathbf{D}_{\mathbf{1}}$ and $\mathbf{D}_{\mathbf{2}}$ are the border points of the reflection.

Using simple trigonometric calculations, position of the points $\mathbf{I}_{\mathbf{1}}$ and $\mathbf{I}_{\mathbf{2}}$ can be expressed as

$$
\begin{aligned}
D_{1 b} & =M_{1 b}-\left(M_{1 z}-I_{1 z}\right) \tan (2 \beta+\gamma), \\
D_{2 b} & =M_{2 b}-\left(M_{2 z}-I_{2 z}\right) \tan (2 \beta+\gamma), \\
\beta & =\alpha-\gamma, \\
\alpha & =\arctan \left(\frac{M_{2 b}-M_{1 b}}{M_{2 z}-M_{1 z}}\right) .
\end{aligned}
$$

As the mirror is supposed to have a finite thickness, it is necessary to test, which side of the mirror reflects. If $\beta<0$ then beams hit the oposite side of a mirror and symbols $\mathbf{M}_{\mathbf{1}}$ and $\mathbf{M}_{\mathbf{2}}$ in eqs. 4-5 have to be replaced by symbols $\mathbf{M}_{3}$ and $\mathbf{M}_{4}$. 


\subsection{Shading between mirrors}

In the calculation of the reflections, one important correction have to be made, since reflection can be shaded by an adjacent mirror. The situation is shown in Fig. 4, where the whole surface between points $\mathbf{M}_{\mathbf{1}}$ and $\mathbf{M}_{\mathbf{2}}$ does not act in the reflection. Only the effective area bordered by the points $\mathbf{M}_{\mathbf{1}}^{\prime}$ and $\mathbf{M}_{\mathbf{2}}^{\prime}$ have to be taken into account. In the detail, this procedure will be specified only for the beams parallel to the $z$-axis. Positions of the

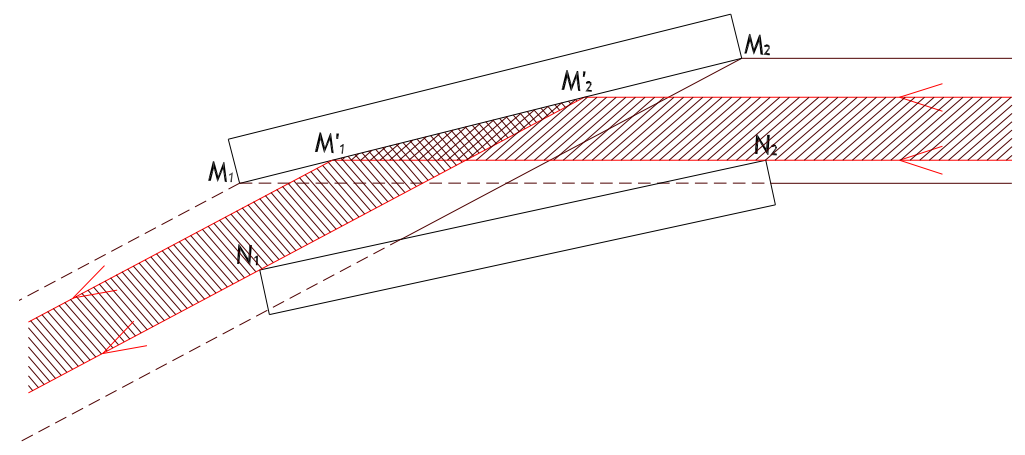

Figure 4. Effect of shading by adjacent mirror

points $\mathbf{M}_{\mathbf{1}}^{\prime}$ and $\mathbf{M}_{\mathbf{2}}^{\prime}$ are calculated using the equations

$$
\begin{aligned}
t & =\frac{M_{2 b}-M_{1 b}}{M_{2 z}-M_{1 z}}, \\
q_{1} & =M_{1 b}-t M_{1 z}, \\
q_{2} & =N_{1 b}-2 t N_{1 z}, \\
M_{1 z}^{\prime} & =\frac{N_{2 b}-q_{1}}{t}, \\
M_{1 b}^{\prime} & =M_{2 b}, \\
M_{2 z}^{\prime} & =\frac{q_{1}-q_{2}}{t}, \\
M_{2 b}^{\prime} & =t M_{2 z}^{\prime}+q_{1} .
\end{aligned}
$$

If $M_{1 z}^{\prime}>M_{2 z}$ or $M_{2 z}^{\prime}<M_{1 z}$ or $M_{1 z}^{\prime}>M_{2 z}^{\prime}$ then the arrea bordered by points $\mathbf{M}_{\mathbf{1}}$ and $\mathbf{M}_{\mathbf{2}}$ is shaded completely and the corresponding foil does not contribute to reflection at all.

If $M_{1 z}^{\prime}<M_{1 z}$ then the theoretical position of the point $\mathbf{M}_{\mathbf{1}}^{\prime}$ lays outside the mirror and the point $\mathbf{M}_{\mathbf{1}}$ is not shaded, this correction for the point $\mathbf{M}_{\mathbf{1}}$ is not used and the position of the point $M_{1}$ itself has to be used for calculations.

Similarly, if $M_{2 z}^{\prime}>M_{2 z}$ then the theoretical position of the point $\mathbf{M}_{2}^{\prime}$ lays outside the mirror and the point $\mathbf{M}_{2}$ is not shaded and the position of the point $\mathbf{M}_{\mathbf{2}}$ itself is used for calculations.

\subsection{Calculation of reflection coefficient}

Reflectivity data for given material (gold) and energies were tabulated ${ }^{12}$ for various grazing angles. Between the given points, the linear interpolation was performed.

\section{COMPARISON OF EXPERIMENTALLY OBTAINED VALUES AND THEORETICAL RESULTS}

Experimentally obtained value ${ }^{8}$ of the field of view(FOV) at X-ray energies from 0.28 to $4.5 \mathrm{keV}$ was $2.9 \pm 0.1^{\circ}$. This value is in good agreement with the value $2.8^{\circ}$ following from the theoretical assumptions ${ }^{7}$. 
Ideally $^{1}$, X-rays are focussed to the spot of size roughly equal to the size of the LE chamber which was $300 \times$ $300 \mu \mathrm{m}$. So, gain was estimated as the measured ratio between the flux incoming to LE and the measured average flux of X-rays measured in the focus region of size $300 \times 300 \mu \mathrm{m}$ (the paper with details about the experiment is in $\left.\operatorname{press}^{8}\right)$. Computer simulation was done with the same assumptions. Comparison of the experimentally given values with the results of simulations is given in Fig. 5 and Tab. 1.

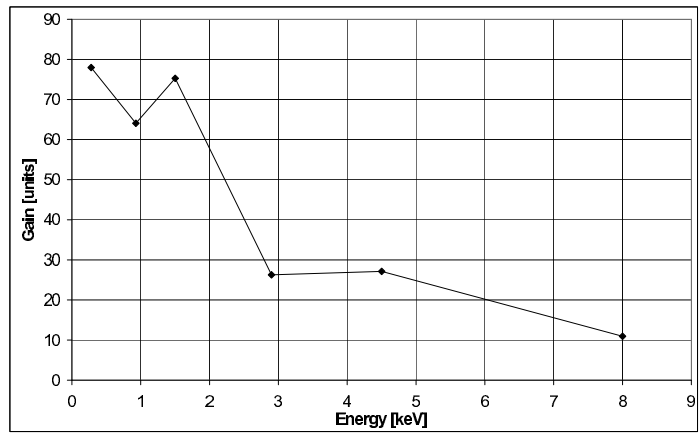

(a) Experiment

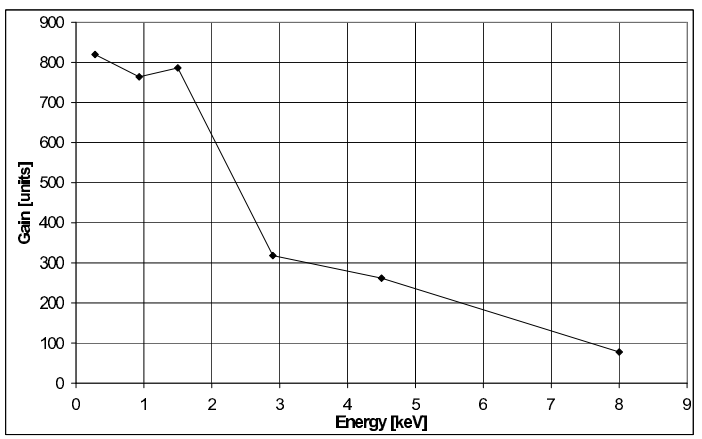

(b) Simulation

Figure 5. Gain of lobster eye P-25 at various energies

\begin{tabular}{|c|c|c|}
\hline Energy [keV] & measured gain & simulated gain \\
\hline 0.28 & $78 \pm 2$ & 820 \\
0.93 & $64 \pm 1$ & 764 \\
1.5 & $75 \pm 1$ & 786 \\
2.9 & $26 \pm 1$ & 318 \\
4.5 & $27 \pm 1$ & 262 \\
8.0 & $11 \pm 1$ & 78 \\
\hline
\end{tabular}

Table 1. Gain of P-25 at various energies

Results show that this lobster eye cannot be effectivelly used for energies higher than approx. $2 \mathrm{keV}$ due to $M_{a 1}$ absorption line of gold laying at the energy $2.1 \mathrm{keV}^{12}$. This fact represents a principal limit for the selection of scientific goals as well as for the selection of a detector used with this lobster eye.

Experimentally obtained ${ }^{8}$ value of the spatial resolution at all X-ray energy values from 0.28 to $8 \mathrm{keV}$ was $13 \pm 1^{\prime}$ (arcmin). This value is also worse then the theoretical ${ }^{7}$ value $5.5^{\prime}$ calculated for ideally assembled lobster eye.

Differences between the theoretical and the simulated values can be explained by two facts related to the manufacturing process. At first, mirrors have not been located to the ideal positions. The manufacturer, Rigaku Innovative Technologies Europe, s.r.o. cannot present details about used technology, but it provided information of the reached accuracy of the settling of the mirrors. The root of mean square error of the deviation of the mirror spacing is approx. $2.5 \mu \mathrm{m}$ (note, this is information about the technology used in this, few years old lobster eye and the current technology promises higher accuracy). This error has the cumulative character, i.e. if one mirror is settled with some deviation, the following mirror is settled with the same deviation plus the deviation of its own settling. In the simulation program, these deviations were simulated. The resulting gain was decreased two- to five-times. Second key manufacturing challenge is the flatness of the mirrors. Sure, mirrors are never ideally flat and their deflections cause decreasing of the gain. The deflection of the mirrors reaches the order of the tens of microns. We can suppose, this fact causes following higher decreasion of the gain. However, this fact cannot be simulated by the program using the presented algorithm and the exact proof that this fact causes the decrease of the gain will be the subject of further research (here, we should also note that the mirrors of much higher flatness are planned to use in future lobster eye specimens). 
The problem is seen in Fig. 6, where the experimentally obtained image of the parallel beam of energy $930 \mathrm{eV}$ is compared with the result of simulation.

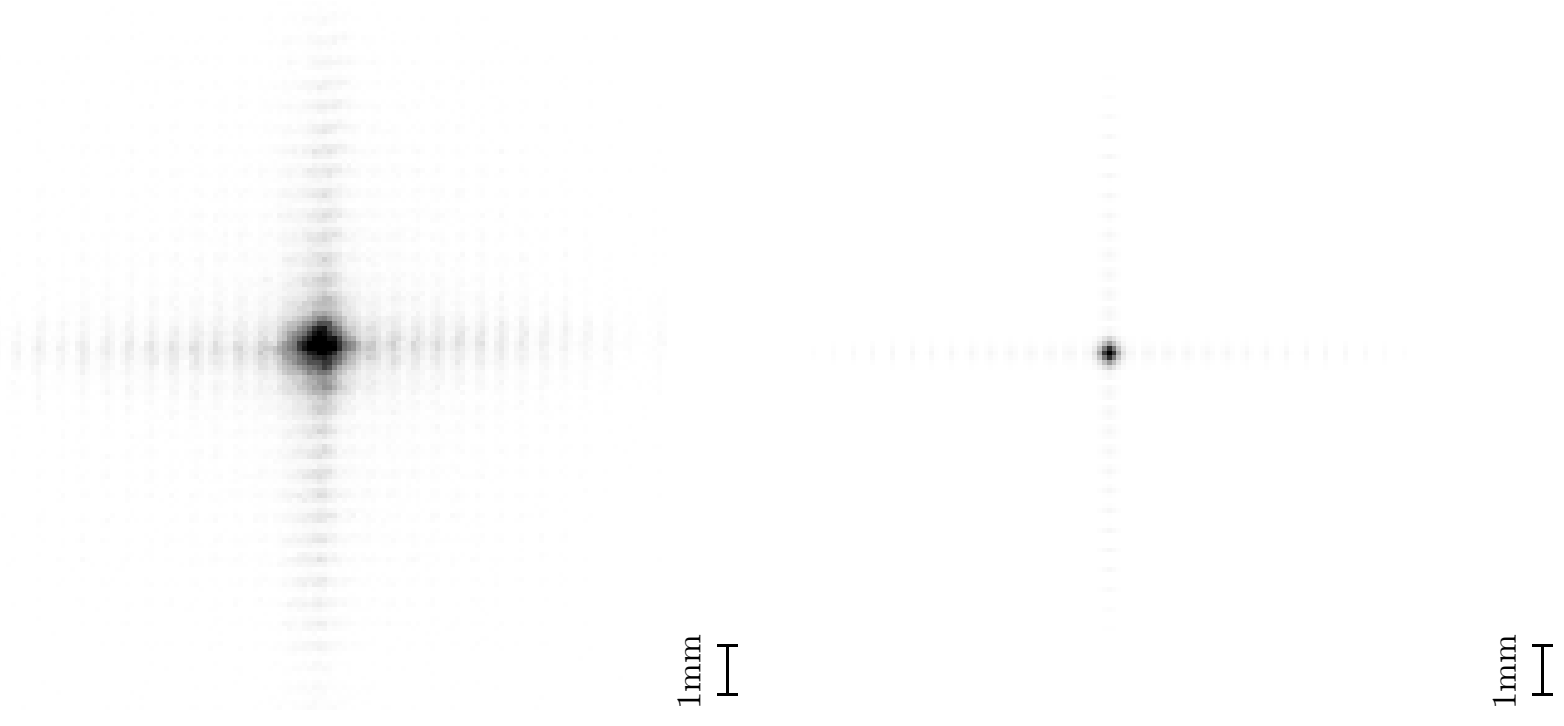

(a) Experiment

(b) Simulation

Figure 6. Comparison of the experimentally obtained focal cross with results of simulation for lobster eye P-25 at energy $930 \mathrm{eV}$.

In Fig. 6(a), the image is smeared and photons are focussed to a region larger than $300 \times 300 \mu \mathrm{m}$.

The lower measured values of the gain are also partially caused by the fact, that the central position found as the center between the corner position is not the position of the maximal gain ${ }^{8}$.

\section{ASSESMENT OF USAGE IN AN ASTRONOMICAL MISSION}

\subsection{Sensitivity assesment}

Measured values of gain are similar at all measured lines $0.28-1.5 \mathrm{keV}$ and the analysis will be made at energy $930 \mathrm{eV}$.

It was shown the the measured image has the spot larger than the projection of the central chamber of the LE, so for the estimation of the sensitivity, real size of the spot is used. The peak has roughly square shape ${ }^{1}$. Measured FWHM size of the peak is approx. $1 \mathrm{~mm}$ at all used energies ${ }^{8}$.

If the gain is estimated from the box of the corresponding size, i.e. of the size $1 \mathrm{~mm} \times 1 \mathrm{~mm}$, the gain at energy $930 \mathrm{eV}$ reaches 38 .

This value is worser than the value previously obtained. However, the key value of the optics to assess its effectivity is not the gain but the effective collecting area $S_{e}$. If the gain $G$ and the spot area $S_{s}$ are known, the effective collecting area can be expressed as $S_{e}=G S_{s} \doteq 0.38 \mathrm{~cm}^{2}$. In the following text, we show this value is sufficient for some astronomical measurements.

\subsection{Space mission assumptions}

\subsubsection{Spacecraft}

Although it is supposed the future lobster eye models will be used in large space missions, as one of the first steps we propose the nano-satellite mission. Due to the focal length of the lobster eye P-25 which is $250 \mathrm{~mm}$, we suppose to use $3 \mathrm{U}$ size, i.e. $30 \times 10 \times 10 \mathrm{~cm}^{3}$ CubeSat $^{11}$ spacecraft. 


\subsubsection{Lobster eye}

As said, we will suppose manufacturing of the larger lobster eye of total collecting area around $8 \times 8 \mathrm{~cm}^{2}$ and focal length still around $250 \mathrm{~mm}$. These parameters should allow to reach total field of view of $5 \times 5^{\circ}$. Because measured field of view is in accordance with the theory and it is simply given by the LE geometry, ${ }^{7}$ we can suppose the assumed FOV $5 \times 5^{\circ}$ would be reached.

Although we expect higher value of the effective collecting area (and consequetly of the sensitivity), to be conservative, we consider the measured value. Likewise, we will suppose the angular resolution to be around 13 arcmin.

\subsubsection{Detector}

Soft X-ray imaging detectors used in space missions lay in one of these three families

- Semiconductor detectors

- Gas detectors

- Microchannel plates (MCP)

Semiconductor detectors require cooling to be able to detect energies around $1 \mathrm{keV}$ and below. Gas detectors require gas supply. The cooling as well as the gas supply represent complication and maybe, it can not be realised respecting the CubeSat limits. So, in this preliminary design proposal we will suppose using of MCP. It can provide sufficient spatial resolution in order of $100 \mu \mathrm{m}$ and sufficient active area (centimeters). Detection efficiency can be up to $30 \%$. Using of another detector will be subject to further investigation.

\subsection{Required minimal flux}

No detailed backround analysis has been performed. We suppose the source is detected if at least 100 photons are focussed. With the mentioned effective collecting area of $0.38 \mathrm{~cm}^{2}$ and considering $30 \%$ quantum efficiency of the detector, the resulting minimal flux to be detected is approx. $1.4 \times 10^{-9} \mathrm{erg} \mathrm{cm}^{-2} \mathrm{~s}^{-1} \approx 60 \mathrm{mCrab}$ with 1 ks exposure time.

\section{PROPOSED SCIENTIFIC GOALS}

Generation of X-ray emission is a natural product of accretion of matter onto the compact objects (white dwarfs (WDs), neutron stars (NSs), black holes (BHs)) in binary systems (cataclysmic variables, X-ray binaries of various types). Accretion process and/or the processes on the surface of the compact object are the dominant sources of X-ray luminosity. X-ray emission of these systems undergoes significant changes on various timescales, from the very fast ones (fraction of second), through the medium fast (days), to the long timescales (years, decades), not speaking about the evolutionary processes. Reviews can be found in e.g. ${ }^{15,17,23}$.

The long-term variations of X-ray emission are poorly understood mainly because of the lack of a sufficiently long data coverage caused by the fact that the observing strategies of most existing and previous satellites only concentrated on the pointing to a sample of objects, with a limited observing time allocated for a given object (typically at most several days). Although this strategy enables studies of faint sources with high temporal and/or spectral resolution, the information of the long-term processes is lacking. Our understanding of the physical processes in a given object or a category of objects cannot be considered to be plausible if the investigation of the physical processes governing the long-term activity is not carried out.

Soft X-rays (with energies less than $2 \mathrm{keV}$ ) in which the proposed lobster eye telescope is most sensitive are very suitable for monitoring since the spectra of most sources that we propose to observe display their peak in this soft band, despite interstellar extinction. Generally, this spectral region contains blackbody component (inner disk region in X-ray binaries, the surface of the compact object), emission via inverse Compton process (Comptonizing cloud in X-ray binaries), bremsstrahlung emission (boundary layer in cataclysmic variables). 
We argue that monitoring of a large part of the sky is needed, not only monitoring of (hence pointing to) the already known objects. The reason is that most transients (e.g. objects with outbursts) were discovered only in outburst, not in quiescence before this event. A lot of 'sleeping' transients of various types thus exist.

The amplitude of X-ray outburst can be more than an order of magnitude (in transients even more than 100). This suggests that we can obtain meaningful information even by a study of faint objects whose low X-ray intensitites are affected by a noticeable uncertainty. Since the large-amplitude variations often occur on the timescale of weeks and months, it is possible to concentrate on the time segments of high luminosity when the observations with a high signal-to-noise $(\mathrm{S} / \mathrm{N})$ ratio are available. Steep rising branches of some outbursts can be an exception. The segments of lower luminosity can be investigated after a binning of the data in time. This binning will enable us to increase the $\mathrm{S} / \mathrm{N}$ ratio.

We briefly discuss the perspectives of analysis of the X-ray data obtained with the proposed lobster eye monitor onboard the nanosatellite. We also discuss the choice of the most promising area of the sky and the types of objects.

The available state of art of high-energy astrophysics ${ }^{15,17,23}$ enables us to conclude that systems containing a mass-accreting NS or BH (the so-called X-ray binaries) are the most promising objects to be detected by our nanosatellite. Accretion onto these compact objects leads to a sufficiently high X-ray luminosity to make them detectable by the nanosatellite at least in some phases of their long-term activity. We therefore briefly list the types of X-ray binaries. This list is based on the books ${ }^{15,17,23}$.

Low-mass X-ray binaries (LMXBs) - systems consisting of a compact object (NS or BH) and a Roche lobe filling donor. The donor is usually a late type main-sequence star, but also rare cases where it is a subgiant, giant or a WD are known (depending on the orbital period ranging from about $11 \mathrm{~min}$ to several days (rarely months), mostly several hours ${ }^{20}$. LMXBs are often highly variable on various time scales, from seconds to decades. They display various kinds of activity, like high/low state transitions or outbursts (see below). Some of them also display the orbital modulation or eclipses of the accretion disk by the donor. LMXBs are generally classified either as persistent or transient X-ray sources. Transient sources are observed to display occasional outbursts, during which their X-ray flux rises typically by several orders of magnitude for several weeks or months (Fig. 7). These outbursts are separated by intervals of quiescence considerably longer (months to years) than the outbursts themselves. These sources are very faint in quiescence. Their activity is interpreted in terms of the thermal-viscous instability of the accretion disk ${ }^{13}$. Persistent sources are repeatedly detected by X-ray instruments, and their X-ray flux varies by a factor of few (Fig. 8). Almost two hundred LMXBs have been discovered in X-rays in our Galaxy but more of them are hidden because they are in a low X-ray state now. More and more such systems are discovered when their outbursts occur.

High-mass X-ray binaries (HMXBs) - systems consisting of a compact, mass accreting object (NS or $\mathrm{BH}$, rarely also a WD) and an early-type star $(\mathrm{O}, \mathrm{B})$ donating mass (the so-called donor). Basic modes of mass transfer: (a) Roche lobe overflow; (b) radial stellar wind in case of supergiant donors; (c) circumstellar disk in case of a Be main sequence star. Periastron passage of the compact object leads to a periodic variability in some HMXBs, especially if they contain a Be component and have an eccentric orbit (see also below). The usual kinds of X-ray activity are outbursts close to the phase of the periastron passages of the compact object and long-term fluctuations. About a hundred HMXBs have been discovered in X-rays in our Galaxy but it is very probable that more of them are hidden because they are in a low X-ray state now. CI Cam (XTE J0421+560) ${ }^{14}$ can serve as an example of such hidden transients - it showed itself as an X-ray binary by its very powerful X-ray to radio outburst in 1998. It reached the peak intensity of almost 2 Crabs in soft X-ray band during the peak of this outburst.

The systems which undergo prominent large-amplitude variations of intensity are further divided into several types. They are called X-ray transients (XTs). They are a subtype of both HMXBs and LMXBs. Sometimes, only a detailed analysis of the multiband observations of such objects can lead to the determination of the proper type (LMXB or HMXB). They are characteristic by their transient increase of X-ray flux, often by more than an order of magnitude. Some XTs in quiescence may not be detectable in X-rays by the current instruments. 


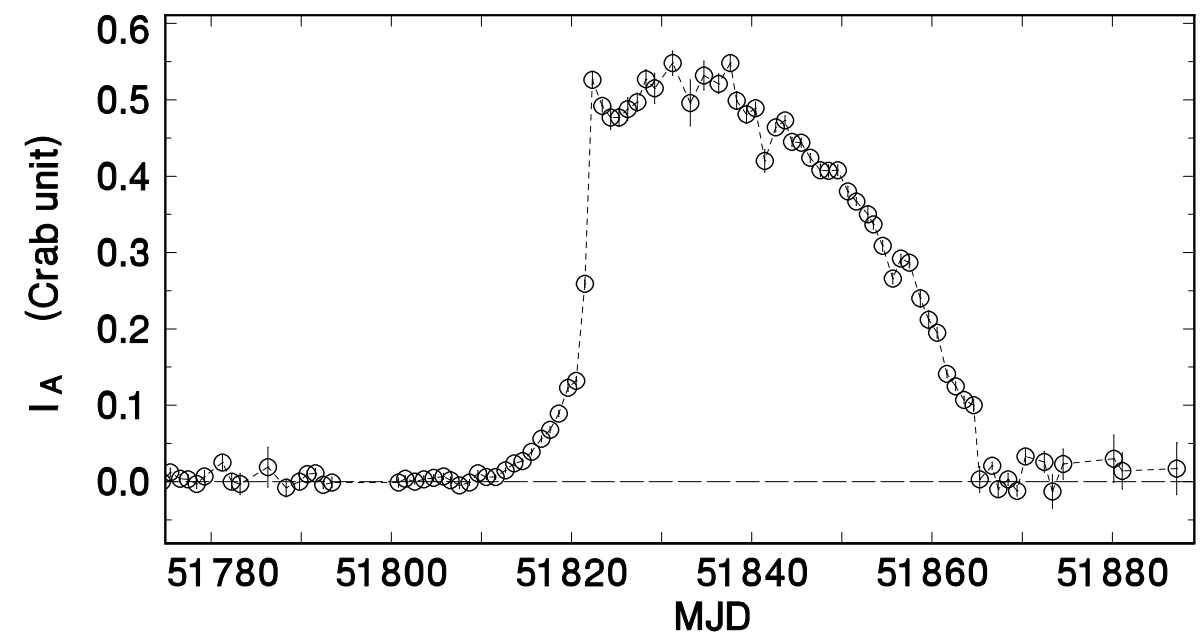

Figure 7. The light curve of a transient source in the $1.5-3 \mathrm{keV}$ band. This band is chosen because it is similar to the band of the peak sensitivity of the proposed lobster eye telescope. The outburst of Aql X-1 observed by ASM/RXTE is taken as an example. Intensities are re-calculated into Crabs.

XTs can be sorted according to the physical processes:

(a) Soft X-ray transients (SXTs), also called X-ray novae, are caused by the thermal-viscous instability of the accretion disk in a LMXB ${ }^{13}$. The physical process is analogous to that in dwarf novae (subtype of cataclysmic variables), but is more energetic and irradiation of the disk by X-rays from the close vicinity of the compact object plays a big role during outburst. The duration of outburst is weeks to months, while the recurrence time (i.e. interval between outbursts) is months to decades. This outburst is defined by a very large increase of X-ray intensity.

(b) Hard X-ray transients - (i) Type I outbursts are dependent on the orbital phase. They occur during periastron passage of the compact object and are attributed to an increase of the mass accretion from the Be donor. This mechanism suggests periodicity but these outbursts need not occur during every passage. The reason is that the amount of the circumstellar matter originating from the Be donor undergoes long-term variations. (ii) Type II outbursts are independent of the orbital phase, i.e. of the position of the compact object on its orbit. They are probably caused by a dramatic expansion of the circumstellar disk.

(c) Very fast X-ray transients - quite a new kind of transient, ${ }^{21}$ only a few are known yet. Each outburst, yet observed only in X-rays, lasts for several hours. These events can be relatively frequent (the recurrence time is of days in some cases). They seem to occur in some HMXBs but the outburst mechanism is uncertain.

Microquasars - X-ray binaries that possess relativistic jets ${ }^{16}$, i.e. collimated outflows of matter moving at relativistic velocities from the compact component (e.g. V4641 Sgr= SAX J1819.3-2525) ${ }^{22}$. Some are HMXBs, the other ones are LMXBs. Some of them display large-amplitude outbursts in various spectral bands, from far X-rays through optical to radio (e.g. V4641 Sgr, CI Cam). The intense 1998 outburst of CI Cam lasted about 2 days in X-rays.

As noted above, transitions between the activity states (e.g. outbursts, high/low states) are often unpredictable. Monitors with wide field of view are thus needed to detect them and to distinguish their profiles. Moreover, even if one concentrates on the already known objects, it emerges that occasional pointing is not enough due to the following reasons. Many pieces of information on the time evolution are lost without long and relatively dense (e.g. once a day or several times a week) time coverage. Also allocation of the observing time with a satellite has to be justified (search for unexpected behavior of the object is usually not approved). 


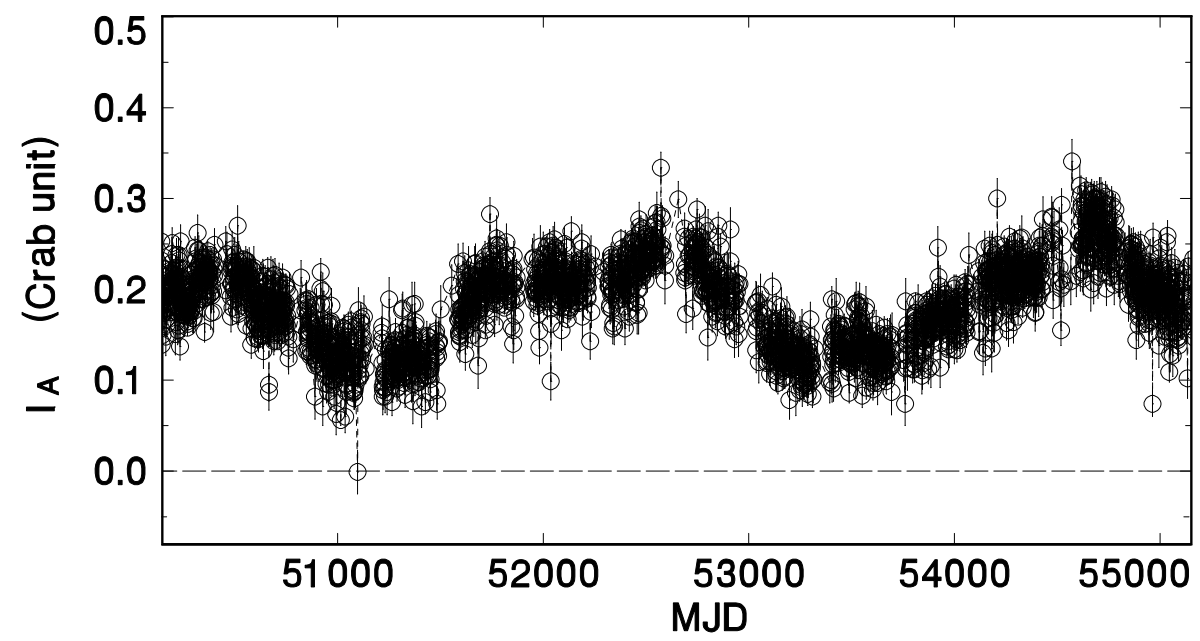

Figure 8. The light curve of a persistent source in the $1.5-3 \mathrm{keV}$ band. This band is chosen because it is similar to the band of the peak sensitivity of the proposed lobster eye telescope. The activity of GX $3+1$ observed by ASM/RXTE is taken as an example. Intensities are re-calculated into Crabs.

The systems that undergo outbursts are particularly promising targets for our monitoring. Even features in the outburst X-ray light curves can be observed by our telescope since the outburst often lasts for several days, sometimes even for several months (Fig. 7). Even observations with several days long gaps and/or strong binning due to a low signal are thus very valuable for our study of the properties of many outbursts.

A lot of results can be achieved even by investigating the profiles of the X-ray light curves obtained in a single band, i.e. without using hardness ratios or spectra. The parameters of irradiation of the accretion disk (e.g. dimensions and luminosity of the irradiating body, profile of the disk), evolution of the irradiating body during outburst, evolution of spiral arms in the disk are predicted to influence the profile of the light curve of outburst, hence they can be studied by our monitor.

In summary, all the above-listed items emphasize the very important role of X-ray monitors in our understanding of the processes operating in X-ray sources.

Now, we will discuss the proper field in the sky for pointing the lobster eye telescope. Field of view of our telescope will be $5^{\circ} \times 5^{\circ}$. To assure a sufficiently high $\mathrm{S} / \mathrm{N}$ ratio, it is desirable to concentrate on monitoring of only a relatively small field. Since the nanosatellite can maneuver, it is possible to compose the resulting area of several smaller areas (each having $5^{\circ} \times 5^{\circ}$ ) covered by the field of view of our telescope. Choosing the proper field of the sky which contains sufficient number of X-ray sources is important for the success of our proposed mission.

The catalogues of LMXBs ${ }^{18}$ and HMXBs ${ }^{19}$ show that the distribution of both LMXBs and HMXBs in the sky is highly anisotropic (Fig. 9). Both types of object concentrate at the Galactic plane. In addition, a concentration toward the center of the Galaxy is prominent, especially for LMXBs. It therefore appears very advantageous to point our telescope toward the Galactic center. We propose the field of $5^{\circ} \times 20^{\circ}$ centered on the center of the Galaxy (Fig. 9). The monitored field will be oriented with its longer axis in the Galactic plane. This represents 4 fields of the lobster eye telescope in the line (or 5 partly overlapping fields to obtain a higher sensitivity this sensitivity decreases with distance from the optical axis of the telescope). This resulting field contains 58 known LMXBs included in the catalogue ${ }^{18}$ and several HMXBs. Many LMXBs are transient sources. It means that they are bright X-ray sources for some time, but their brightenings are recurrent. In addition, many new transients appear in outburst. Usually, only such a new outburst enables us to discover them.

Of course, it is quite likely that also other types of object will be bright enough to be detected by our proposed lobster eye. For example, cataclysmic variables are the candidates. However, their X-ray luminosities are usually 


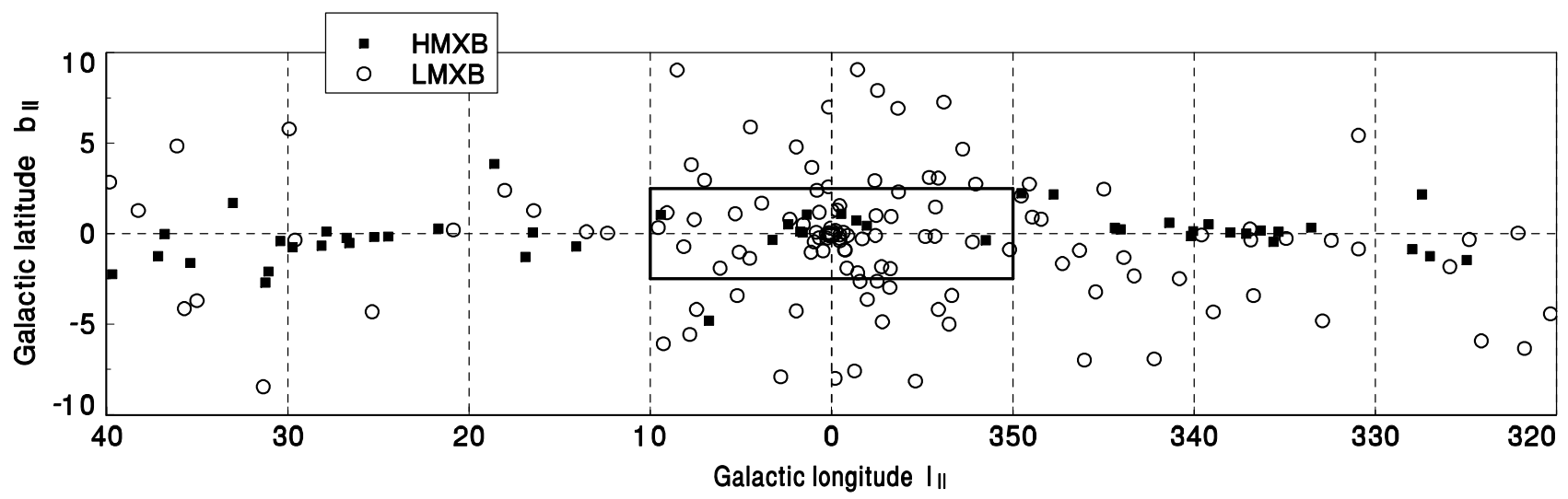

Figure 9. The field of the center of the Galaxy $\left(20^{\circ} \times 80^{\circ}\right)$. The positions of known LMXBs and HMXBs are marked. The field proposed for the monitoring by lobster is marked by the oblong. It contains a number of the already known LMXBs and HMXBs.

lower than those achieved by LMXBs and HMXBs. They are thus not the primary candidates at this phase of our research.

It is also important to note that it will be possible to co-add the individual images. This procedure will enable us to find much fainter objects, albeit with heavily smoothed light curves.

\section{CONCLUSIONS}

Although we reported differences between the theoretical and measured values, presented results still indicate that the proposed lobster eye nano-satellite mission is feasible using known and verified technologies.

Results of imaging tests of the introduced lobster eye specimen were compared to the results of the simulations or theoretical equations. Measured value of the field of view corresponds to the theoretical value. Measured values of gain are approx. 10 times worse than the results of the simulations at all used energies from $0.28 \mathrm{keV}$ to $8.0 \mathrm{keV}$, meaured spatial resolution is 2.4-times worse than the theoretical prediction. These differences are explained by the manufacturing deviations.

Although measured gain and spatial resolution are many times worse than their theoretical values, we showed the achieved results are sufficient for monitoring of some specific astronomical targets and allow to obtain scientifically significant data. Especially, low and high mass X-ray binaries (LMXBs and HMXBs) laying in the center of the Galaxy seem to be good targets for the observations. Detailed scientific mission plan will be the subject of the further research.

Moreover, it can be expected that the performances of the lobster eye will be improved using the current manufacturing technologies, using lobster eye with larger collecting area and using specialised data-processing method.

Detailed proposal of the whole spacecraft and telescope design with respect also to the power, mass and all other limitations of the CubeSat satellite will be the subject of the detailed technological study.

\section{ACKNOWLEDGMENTS}

Authors would like to thank Prof. Salvatore Sciortino, director of INAF-Osservatorio Astronomico di Palermo for providing the use of the X-ray beam-line for the experiments. Thanks to Rigaku Innovative Technologies Europe s.r.o. for providing of the lobster eye specimen.

Thanks for the financial support from the following grants: MSMT 1M0567, MSMT KONTAKT No. ME10094, MSMT ME0918, MSMT ME09028, MSM 6840770022, ME 09004. 


\section{REFERENCES}

[1] W. H. K. Schmidt, "A proposed X-ray focusing device with wide field of view for use in X-ray astronomy", Nucl. Instr. Meth. 127, 285-292 (1975)

[2] J. R. P. Angel, "Lobster eyes as X-ray telescopes", ApJ 233, 364-373 (1979)

[3] R. Hudec, A. Inneman, L. Pína, V. Hudcová, L. Švéda, H. Tichá, "Lobster eye x-ray telescopes: recent progress", Proc. SPIE 4851, 578-4851 (2003)

[4] R. Hudec, L. Pína, V. Šimon, L. Švéda, A. Inneman, V. Semencová, M. Skulinová, "LOBSTER New Space XRay telescopes", Nucl. Phys. B - Proc. Suppl. 166, 229-233 (2007)

[5] http://heasarc.gsfc.nasa.gov/docs/xte/XTE.html

[6] http://xte.mit.edu

[7] V. Tichý, M. Hromčík, R. Hudec, A. Inneman, J. Maršík, V. Maršíková, L. Pína, ”Tests of Lobster-Eye Optics for a Small X-Ray Telescope", Balt. Astr. 18, 362-368 (2009)

[8] V. Tichý, M. Barbera, A. Collura, M. Hromčík, R. Hudec, A. Inneman, J. Jakůbek, J. Maršík, L. Pína, V. Maršíková, L. Pína, S. Varisco, "Tests of Lobster Eye Optics for Small Space X-ray Telescope", Nucl. Instr. Meth. A, in press, available on-line at http://dx.doi.org/10.1016/j.nima.2010.06.157

[9] M. Barbera, R. Candia, A. Collura, G. DiCicca, C. Pelliciari, S. Sciortino, S. Varisco, "The Palermo XACT facility: a new $35 \mathrm{~m}$ long soft X-ray beam-line for development and calibration of next generation X-ray observatories", Proc. SPIE 6266, F2663-F2663 (2006)

[10] http://www.astropa.unipa.it/XACT/index.html

[11] http://www.cubesat.org/

[12] http://henke.lbl.gov/optical_constants/

[13] G. Dubus, J.-M. Hameury, J.-P. Lasota, "The disc instability model for X-ray transients: Evidence for truncation and irradiation", $A \& A$ 373, 251-271 (2001)

[14] F. Frontera, M. Orlandini, L. Amati et al., "Multifrequency observations of XTE J0421+560/CI Cam in outburst", $A \& A$ 339, L69-L72 (1998)

[15] W. H. G. Lewin, J. van Paradijs, E. P. J. van den Heuvel, X-ray binaries, Cambridge Astrophysics Series, Cambridge University Press, Cambridge (1995)

[16] I. F. Mirabel, L. F. Rodríguez, "Sources of Relativistic Jets in the Galaxy", ARA EA 37, 409-443 (1999)

[17] W. H. G. Lewin, M. van der Klis, Compact stellar X-ray sources, Cambridge Astrophysics Series, Cambridge University Press, Cambridge (2006), ISBN 978-0-521-82659-4

[18] Q. Z. Liu, J. van Paradijs, E. P. J. van den Heuvel, "A catalogue of low-mass X-ray binaries in the Galaxy, LMC, and SMC (Fourth edition)", A\&A 469, 807-810 (2007)

[19] Q. Z. Liu, J. van Paradijs, E. P. J. van den Heuvel, "Catalogue of high-mass X-ray binaries in the Galaxy (4th edition)", $A \& 3 A$ 455, 1165-1168 (2006)

[20] H. Ritter, U. Kolb, "Catalogue of cataclysmic binaries, low-mass X-ray binaries and related objects (Seventh edition)", A\&SA 404, 301-303 (2003)

[21] V. Sguera, E. J. Barlow, A. J. Bird et al., "INTEGRAL observations of recurrent fast X-ray transient sources", A\&̈A 444, 221-231 (2005)

[22] R. Stubbings, IAUC 7253 (1999)

[23] B. Warner, Cataclysmic Variable Stars, Cambridge Univ. Press, Cambridge (1995) 